\title{
The contribution of the pericanalicular matrix to mineral content in human osteonal bone.
}

\author{
*A. Roschger ${ }^{1,2,}, \mathrm{PhD}$ \\ P. Roschger ${ }^{2}$, PhD, Assoc. Prof \\ W. Wagermaier ${ }^{1}, \mathrm{PhD}$ \\ J. Chen ${ }^{1,3}, \mathrm{PhD}$
}

A. van $\mathrm{Tol}^{1}, \mathrm{MSc}$
F. Repp ${ }^{1}, \mathrm{PhD}$
S. Blouin ${ }^{2}, \mathrm{PhD}$

A. Berzlanovich ${ }^{4}$, Prof. MD

G. M. Gruber ${ }^{5}$, PhD

K. Klaushofer², Prof. MD

P.Fratzl ${ }^{1}, \mathrm{PhD}$, Prof

R. Weinkamer ${ }^{1}, \mathrm{PhD}$

${ }^{1}$ Max Planck Institute of Colloids and Interfaces, Department of Biomaterials, 14476 Potsdam, Germany

${ }^{2}$ Ludwig Boltzmann Institute of Osteology at the Hanusch Hospital of WGKK and AUVA Trauma Centre Meidling, $1^{\text {st }}$ Medical Department, Hanusch Hospital, Heinrich Collin Str. 30, A-1140 Vienna, Austria

${ }^{3}$ College of Engineering, Mathematics, and Physical Science, University of Exeter, Exeter EX4 $4 \mathrm{QF}$,

${ }^{4}$ Department of Forensic Medicine, Medical University of Vienna, Sensengasse 2, A-1090 Vienna, Austria

${ }^{5}$ Department of Anatomy, Center for Anatomy and Cell Biology, Medical University of Vienna, A-1090 Vienna, Austria

*corresponding author, Andreas.Roschger@sbg.ac.at, 004366280446205

${ }^{\circ}$ current address, Paris-Lodron University of Salzburg, Department of Chemistry and Physics of Materials, 5020 Salzburg, Austria

The research presented here was supported by a grant from the German Federal Ministry of Education and Research (Grant No. 01EC1402D) and by the AUVA (Research Funds of the Austrian Workers Compensation Board, Austria) and the WGKK (Viennese sickness insurance funds, Austria).

In addition to the main manuscript a movie will be provided Online as Supplementary Information.

Disclosure

The authors have nothing to declare, there is no conflict of interest. 


\begin{abstract}
The osteocyte lacunar-canalicular network (LCN) penetrates bone and houses the osteocytes and their processes. Despite its rather low volume fraction, the LCN represents an outstanding large surface that is possibly used by the osteocytes to interact with the surrounding mineralized bone matrix thereby contributing to mineral homeostasis. The aim of this study was to quantitatively describe such contributions by spatially correlating the local density of the LCN with the mineral content at the same location in micrometer-sized volume elements in human osteons. For this purpose, 65 osteons from the femur midshaft from healthy adults $(n=4)$ and children $(n=2)$ were structurally characterized with two different techniques. The 3D structure of the LCN in the osteons was imaged with confocal laser scanning microscopy after staining the bone samples with rhodamine. Subsequent image analysis provided the canalicular length density, i.e. the total length of the canaliculi per unit volume $\left(\mu \mathrm{m} / \mu \mathrm{m}^{3}\right)$. Quantitative information on the mineral content $(w t \% \mathrm{Ca})$ from the identical regions was obtained using quantitative backscattered electron imaging.

As the LCN-porosity lowers the mineral content, a negative correlation between Ca content and network density was expected. Calculations predict a reduction of around $-0.97 \mathrm{fmol}$ Ca per $\mu \mathrm{m}$ of network. However, the experiment revealed for 62 out of 65 osteons a positive correlation resulting in an average additional $\mathrm{Ca}$ loading of $+1.15 \mathrm{fmol}$ per $\mu \mathrm{m}$ of canalicular network, i.e. an accumulation of mineral has occurred at dense network regions. We hypothesize that this accumulation happens in the close vicinity of canaliculi forming mineral reservoirs that can be utilized by osteocytes. Significant differences found between individuals indicate that the extent of mineral loading of the reservoir zone reflects an important parameter for mineral homeostasis.
\end{abstract}

Keywords:

Osteocytic osteolysis, osteocyte network, canaliculi, mineralization, osteons, osteocyte 


\section{INTRODUCTION}

The osteocyte network in bone is an intriguing example of how living cells interact with their surrounding environment. Osteocytes are cells housed in lacunae inside the mineralized bone matrix ${ }^{(1,2)}$. They originate from osteoblasts, which get encased in the matrix in the course of bone formation. During this process, early osteocytes develop processes interconnecting them with their neighbors and with lining cells at the bone surface. In the maturated mineralized bone matrix, the cell bodies of the osteocytes are located in cavities called osteocyte lacunae which radiate in around $300 \mathrm{~nm}$ wide canals, the canaliculi, that house the dendritic processes ${ }^{(3)}$. This so-called osteocyte lacunar-canalicular network (LCN) pervades all bones of mammals displaying a sophisticated geometry that differs between species ${ }^{(4)}$, skeletal sites ${ }^{(5)}$, loading and health conditions ${ }^{(6)}$, age ${ }^{(7,8)}$ and is linked to the orientation of the surrounding collagen matrix ${ }^{(4,5,9,10)}$. The primary role of osteocytes and the LCN for bone health is related to its multiple functions ${ }^{(1,2)}$. It contributes to the strain-amplification mechanism for mechanosensing ${ }^{(11,12)}$ and provides an infrastructure for efficient nutrition and endocrine transport ${ }^{(1,11,13)}$. Recently, there is growing evidence of an important role of the osteocytes in mineral homeostasis using processes referred to as osteocytic osteolysis or perilacunar/pericanalicular remodeling ${ }^{(14-16)}$. Despite the relatively low volume fraction of the LCN (around 2\%), geometrical considerations already point in this direction since the total surface area of the LCN in an adult is estimated to be around $215 \mathrm{~m}^{2}(17)$. This is much larger than the outer bone surface accessible to osteoclasts and osteoblasts ${ }^{(17)}$, making the LCN more suitable to play an important role in the mineral homeostasis. Further corroboration is based on the observation that $80 \%$ of the mineralized bone matrix is closer than roughly $1.4 \mu \mathrm{m}$ to the LCN in sheep bone ${ }^{(9)}$ and around $3 \mu \mathrm{m}$ in human bone ${ }^{(18)}$. Therefore, changes in mineral content in such narrow regions around the canaliculi potentially provide a significant source of mineral. 
Studies on mice demonstrated that bone matrix was resorbed around lacunae and canaliculi during lactation ${ }^{(19)}$ with a subsequent re-mineralization ${ }^{(20)}$. After parathyroid hormone (PTH) infusion, a fringe region with a diameter of a few micrometer of demineralized bone matrix around canaliculi were also reported in mice ${ }^{(19)}$, while glucocorticoid treatment appears to inhibit perilacunar remodeling ${ }^{(21)}$. It has been speculated that pericanalicular remodeling is caused by acid secretion starting at the osteocyte dendrites. This is in line with reports that osteocytes express markers that are usually attributed to osteoclasts' resorption activities like cathepsin K or tartrate-resistant acid phosphatase (TRAP) ${ }^{(22)}$. However, in rats immobilization and long-term recovery was found not to affect osteocyte lacunar volume or density ${ }^{(23)}$ but estrogen deficiency revealed to alter lacunarcanaliculi microenvironment ${ }^{(24)}$.

In human bone it was observed that canaliculi are confined to narrow regions of loosely packed unordered collagen matrix located between arrays of well-aligned fibrils using scanning and transmission electron microscopy ${ }^{(25)}$. However, second harmonic generation imaging showed that the ordered part of the collagen matrix is coaligned with the preferred orientation of canaliculi in both human and bovine bone ${ }^{(5,10)}$. Using synchrotron phase-contrast tomography, Hesse et al. found regions of increased mineralization around osteocyte lacunae and canaliculi in healthy humans. In the perilacunar and the pericanalicular regions, they observed a peak in mass density while a basal level of mineral content is asymptotically reached with growing distance to the interface with the $\mathrm{LCN}^{(3)}$. However, the extent to which the $\mathrm{LCN}$ might contribute to the overall mineralization of bone remains unknown.

The strategy of the present work is to use a correlative approach to test the hypothesis that the osteocytes' access to the mineralized matrix directly influences the degree of mineralization. For this test we investigate whether network-dense and network-loose regions exhibit different degrees of matrix mineralization by correlating two quantitative measures: (i) the LCN is imaged with 
confocal laser scanning microscopy (CLSM) after rhodamine staining and is characterized by its length density (Can.Dn ${ }^{1}$ ) and (ii) the local calcium (Ca) content of the mineralized matrix is evaluated by quantitative backscattered electron imaging (qBEI) in the corresponding local domains.

In previous studies focusing on adult human osteonal bone, the average network density was determined to be $0.074 \mu \mathrm{m} / \mu \mathrm{m}^{3}$ (i.e., $74 \mathrm{~km}$ per cubic centimeter of bone) exhibiting substantial variations within the osteons and a decrease in network density with distance to the Haversian canal ${ }^{(10,18)}$. To characterize mineral content, over the last two decades, qBEI has been developed as a standard tool to quantify spatial variations in the $\mathrm{Ca}$ content of bone reported as $\mathrm{wt} \% \mathrm{Ca}{ }^{(26)}$.

In the present study, our aim is to detect and to quantify bone mineralization with respect to the local LCN density. The rationale of our investigation is that such a contribution would reveal itself in a spatial correlation between the two quantities, network density and Ca content, evaluated in a multitude of small bone volumes within human osteons of femoral cortical bone of healthy individuals. The detection of a network active in mineral homeostasis is crucially facilitated by a clear baseline defining an inactive, "inert" LCN where matrix mineralization is independent of the network. In this case, the network would manifest itself as locations where bone is simply missing, and consequently the qBEI signal would be lowered proportional to the density of the network. Assuming a typical diameter of the canaliculi, the reference value for an inactive network will be calculated and compared to our measurements. By correlating the results of CLSM and qBEI experiments, we will introduce a new parameter describing the increase/decrease of the local mineral content due to the presence of the LCN as Ca loading per $\mu \mathrm{m}$ of canalicular network.

\footnotetext{
${ }^{1}$ We modified the more common abbreviation "Ca.Dn" to "Can.Dn" in order to avoid confusion with the Ca content.
} 


\section{MATERIAL \& METHODS}

\section{Samples and Sample preparation}

Necropsy samples from the femur midshaft of six female individuals (four adults, two children) without any known bone-related disease were analyzed (see Table 1 for individual age and sex). None of the presented samples or data were presented in previous studies, but three of the four samples from adults originate from the same individuals as used by Repp et al to study the network heterogeneity and co-alignment with collagen ${ }^{(10,18)}$. For our study, $1 \mathrm{~cm}$ thick pieces of the femur midshaft diaphysis were cut out of the frozen femurs transversally (i.e., perpendicular to the long axis of the bone). As the majority of Haversian canals are oriented along the long axis of the femur midshaft, the osteons exhibit an approximately circular shape in our cutting plane. The sample size was further reduced by selecting the lateral side of the cortex as regions of interest. A dehydration

series was performed involving gradually increasing ethanol concentrations ${ }^{(27,28)}$. Prior to polymethylmethacrylate (PMMA) embedding, staining with rhodamine was performed. The rhodamine molecule is small enough $(\sim 0.9 \mathrm{~nm})$ to penetrate the cortical bone via the canaliculi ${ }^{(11)}$ and is known to bind to all inner and outer bone surfaces. In the staining procedure the samples were put into tubes $(50 \mathrm{ml})$ with a mixture of ethanol and rhodamine $(0.417 \mathrm{~g}$ rhodamine $6 \mathrm{G}$ (Acros Orgsnics, Geel - Belgium) / 100ml ethanol). The tubes were mounted overnight on a low-speed spinning wheel to provide optimal penetration of the staining fluid. This procedure was repeated for three times, each time with a fresh ethanol-rhodamine mixture, to provide the highest possible stain concentrations in the sample. Subsequently, PMMA embedding was performed and the blocks were again cut transversally in the middle using a low-speed diamond saw (Buehler Isoment, Lake Bluff, Illinois). The sample surface was ground with sand paper and polished using a diamond suspension (3 and $1 \mu \mathrm{m}$ grain size, Logitech PM5, Glasgow, Scotland). After collecting fluorescent images at a laser scanning confocal microscope as described below, the samples were coated with 
carbon to provide a conducting surface for qBEI without any additional grinding/polishing (Agar SEM Carbon Coater; Agar Scientific, Stansted, UK).

All samples were provided by the Department of Forensic Medicine and the Department of Anatomy of the Medical University of Vienna. The study was performed in accordance with the ethic commission board of this institution (EK no. 1757/2013).

Table 1 Samples

\begin{tabular}{|c|c|c|c|}
\hline Individual & age & Sex & \# of imaged osteons \\
\hline Adult 1 & $55 y$ & F & 10 \\
\hline Adult 2 & $50 y$ & F & 15 \\
\hline Adult 3 & $56 y$ & F & 10 \\
\hline Adult 4 & $48 y$ & F & 15 \\
\hline Child 1 & $2.5 y$ & F & 9 \\
\hline Child 2 & $1.3 y$ & F & 6 \\
\hline \multicolumn{3}{|c|}{ Total } \\
\end{tabular}

\section{Confocal laser scanning microscopy (CLSM) and image analysis}

CLSM was used to visualize the lacunar-canalicular network (LCN) in 3-dimension, followed by automated image analysis and quantification. With a Leica TCS SP5 (Wetzlar, Germany) equipped with an oil immersion lens (Leica, HCX PL APO 40x NA 1.25 OIL) the rhodamine-stained and PMMA embedded bone samples were measured to obtain the rhodamine-fluorescence signal and therefore the LCN architecture in these regions. The $543 \mathrm{~nm}$ line (HeNe-laser) was chosen for rhodamine excitement and the fluorescence signal was measured in a spectral window between 553 and $705 \mathrm{~nm}$ with the airy 1 pinhole of $67.93 \mu \mathrm{m}$ (3D image stacks) or $200 \mu \mathrm{m}$ (2D overview images). Simultaneously, also the confocal reflection signal of the $488 \mathrm{~nm}$ argon-laser line was measured with a separate photomultiplier.

To guide the selection of osteons, firstly, 2D overview images from the sample surface were generated making use of the automated grid scan and stitching routine of the operating software 
(Leica LAS AF v 2.64.872). By stitching up to 1444 single images per sample, a region about 12.5 $\mathrm{x} 12.5 \mathrm{~mm}$ was mapped covering the complete thickness of the femoral cortex. These rhodamine fluorescence overview images were used to select osteons of an approximately circular shape with an intact network covering in the whole cross sectional area of the osteon for further 3D network evaluation (see Table 1 for the number of investigated osteons for each individual).

For the 3D assessment of the $\mathrm{LCN}$, the field of view of the obtained single images $\left(388 \times 388 \mu \mathrm{m}^{2}\right.$, $1024 \times 1024$ pixel resolution, i.e. pixel size $=0.379 \mu \mathrm{m})$ in the image stack was large enough to cover whole osteons. A scan speed of 200 lines per second without averaging was used. The depth of imaging was restricted to about $50 \mu \mathrm{m}$ due to the attenuation of the fluorescent radiation through the fully mineralized sample. To compensate for the loss of signal when scanning along the depth, laser intensity and photomultiplier gain were continuously increased. The z-step size (thickness) of $0.3 \mu \mathrm{m}$ resulted in about 170 images per image stack and region. A similar measurement setup was previously successfully used to gain information about network density and canaliculi orientation in rhodamine-stained non-embedded human osteonal bone samples ${ }^{(4,10,18)}$.

For the network characterization we used an established protocol based on ${ }^{(10,18)}$ : (i) staining the LCN of the bone with rhodamine; (ii) imaging the LCN with confocal laser scanning microscopy (CLSM); (iii) thresholding (binarization) of the raw data; (iv) skeletonization, and (v) network quantification. A key parameter is the Can.Dn which describes the length of the canaliculi per unit bone volume. The evaluation was performed using the open source software TINA ${ }^{(29)}$. What follows is a succinct description of the image analysis steps, more information can be found in references ${ }^{(10,18,30)}$ and on the TINA website. Binarization is done using a local thresholding algorithm based on the difference of Gaussians ${ }^{(31)}$ which led to more robust results than using global thresholding. As lacunae appear as much more bulky objects compared to the narrow canaliculi, bulkiness is used as criterion for distinguishing lacunae and canaliculi. Parameters for 
thresholding and lacunae segmentation are chosen in order to gain reliable and robust results accounting for noise and differences in the staining intensity. All datasets were then evaluated with the same thresholding/segmentation parameter set. After segmentation of the lacunae and thresholding of the images, skeletonization is performed by removing voxels from the structure surface layer by layer as long as the connectivity of the canaliculus is preserved. Hence, canaliculi are then represented as chains of single voxels located at the center of the channels obtained in the raw image structures. Outgoing from this skeleton, a network is generated by converting the pixel structure into nodes (joints of two or more canaliculi) and edges (lines connecting two nodes) as shown in Fig 1. It should be noted that diameters of canaliculi cannot be quantified with the spatial resolution of $400 \mathrm{~nm}$ provided by optical microscopy since their diameters range from 290-440 $\mathrm{nm}$ ${ }^{(32)}$. Therefore, instead of the volume fraction of the LCN, the denseness of the canalicular network is evaluated as the canalicular length density, Can.Dn, which is the total length of all canaliculi per volume of interest $\left[\mu \mathrm{m} / \mu \mathrm{m}^{3}\right]$. The volume of interest to characterize spatial variations of the network density within osteons was chosen to be a cube with a side length of $7.37 \mu \mathrm{m}$, i.e. a volume of $400 \mu \mathrm{m}^{3}$.

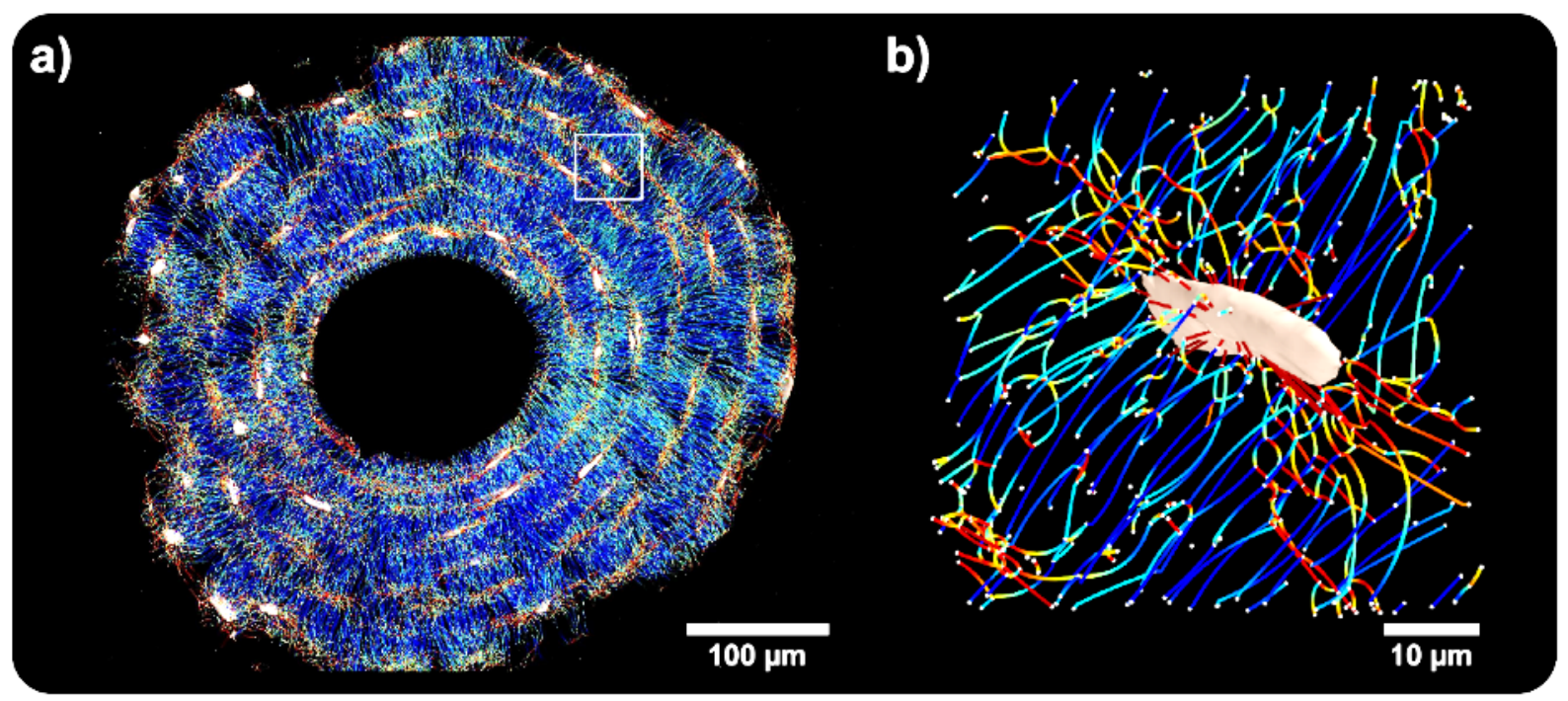

Figure 1: Quantitative description of the lacunar-canalicular network (LCN): After acquisition of the rhodamine fluorescent signal in a confocal laser scanning microscope (CLSM) 3D data are 
segmented by thresholding, skeletonized and converted into a mathematical network consisting of nodes (small white sphere in b) and edges. The color of the edges correspond to their orientation; radial canaliculi pointing towards the Haversian canal are dark blue, lateral canaliculi perpendicular to the radial direction red. a) Shows a typical dataset from an osteon while in b) a small volume (see white box in a) around an osteocyte lacunae and its environment is shown. A movie illustrating the process of data processing is included in the Supplementary Information (see SI-Movie-1).

\section{Quantitative backscattered electron imaging (qBEI):}

Using qBEI, it is possible to quantify the local Ca content of the mineralized bone matrix with a spatial resolution in the micrometer range ${ }^{(28)}$. Backscattered electron images were obtained from the seven femur cross-sections, covering all preselected osteons. The images exhibit a characteristic contrast due to the local average atomic number $(Z)$ of the target material. To calibrate the system, brightness and contrast is adjusted so that in 8-bit images the gray levels of carbon and aluminum reference samples are set to 25 and 225 respectively as described in reference ${ }^{(27)}$. After taking 10 images (about 30 minutes) the calibration samples are revisited to monitor the system stability. If deviations from the calibration values were larger than +-1 gray value, the measurements were repeated. In the calibrated qBEI images, each gray value is assigned to a certain Ca content (weight percent $\mathrm{Ca}$, wt $\% \mathrm{Ca}$ ), where a difference of one gray value corresponds to $0.17 \mathrm{wt} \% \mathrm{Ca}{ }^{(27)}$. qBEI results had been previously validated using energy dispersive $\mathrm{X}$-ray (EDX) analysis and are shown to be consistent with the mineral/matrix ratio obtained with Raman spectroscopy ${ }^{(27,33)}$.

Measurements were performed using a Zeiss Supra40 (Oberkochen, Germany) scanning electron microscope equipped with a ring-like 4-quadrant backscattered electron detector. The acceleration voltage for the electrons was set to $20 \mathrm{kV}$ generating a probe current at the sample surface of about $290 \mathrm{pA}$ at a working distance of $10 \mathrm{~mm}$. 8-bit images of $1024 \times 768$ pixels were taken at $65 \mathrm{x}$ magnification $(1.76 \mu \mathrm{m}$ pixel resolution). According to the literature, the penetration depth at 20 $\mathrm{kV}$ acceleration voltage can be estimated to $1.5 \mu \mathrm{m}$ in a bone-like material ${ }^{(34)}$. Local charging of 
the sample could cause increased local brightness of the backscatter images and consequently corrupt the qBEI outcome. Hence, the secondary-electron mode was used in order to assure that there are no charging effects in the regions of interest.

\section{Superposition of qBEI and CLSM images}

To study the spatial correlation between Ca content and LCN density (Can.Dn) based on small volumes of interest (voxel) within single osteons, it is essential that both parameters are evaluated at the very same locations. As the imaging contrast is different in rhodamine fluorescence and qBEI images, the confocal reflection images from the sample surface (first slices of the simultaneously acquired reflection stack) are used to enable an accurate superposition of the images. The confocal reflection images perfectly match the fluorescent images and have the advantage to sharply exhibit osteocyte lacunae and the Haversian canal; features that are also clearly visualized in the qBEI image. The contour of the Haversian canal and the lacunae can, therefore, be utilized to translate, rotate and scale the qBEI images to precisely match the confocal reflection images (and therefore the fluorescence signal) with an uncertainty of around $1 \mu \mathrm{m}$ as also previously described in reference ${ }^{(35)}$.

For the evaluation of the local network density a cubic evaluation grid of sub volumes with $7.37 \mathrm{x}$ $7.37 \times 7.37 \mu \mathrm{m}^{3}$ was used. The resulting volume of $400 \mu \mathrm{m}^{3}$ is large enough that most of the subvolumes contain canaliculi and is in the order of the size the osteocyte lacunae. The same grid is transferred to the superimposed $\mathrm{qBEI}$ image and the average Ca concentration is calculated for each $7.37 \times 7.37 \mu \mathrm{m}^{2}$ pixel as shown in Fig. 2b). Since electrons in qBEI probe only a surface layer, a correlation with values of the Can.Dn has to be restricted to voxels from the top layer of the 3D 
grid (Fig. 2c). For pixels/voxels containing osteocyte lacunae or located not fully within the mineralized matrix, average values for the Ca content/Can.Dn over a reduced bone volume were calculated to minimize partial volume effects. To evaluate the dependency of the Ca content and Can.Dn as a function of the distance from the Haversian canal, for each pixel/voxel the shortest distance of the center of the pixel to the Haversian canal was determined resulting in a distance map as shown in Fig. 2d.

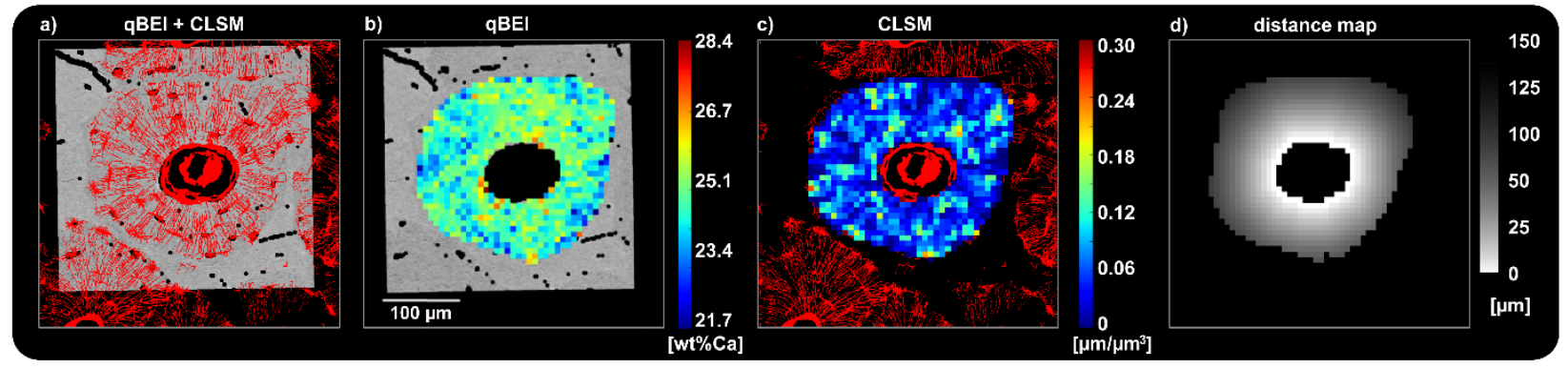

Figure 2: a) qBEI and 3D-CLSM images are superimposed and are aligned along the same grid of $7.37 \mu \mathrm{m}$ voxel/pixel edge size. Mean values of the Ca content (b) and network density (c) are calculated and color coded for each subvolume. (d) To evaluate the radial dependency of the two quantities a distance map is generated where the grey value codes the shortest distance of each pixel to the Haversian canal.

\section{Statistics}

To perform comparisons between the age groups, the Mann-Whitney test was used, while the Wilcoxon signed-rank test was performed to test cohorts of osteonal evaluation parameters vs predefined values. One way ANOVA and subsequent Bonferroni post-hoc tests were applied if multiple datasets had to be compared. Whisker plots indicate median, $25 \%$ and $75 \%$ percentile, and range of the measurements. Significance levels are indicated with different numbers of asterisks and circles and refer to significance levels of $\mathrm{p}<0.05, \mathrm{p}<0.005$ and $\mathrm{p}<0.0001$ respectively. Statistics was performed using GraphPad Prism 5.

\section{RESULTS}




\section{Predicted correlation between $\mathrm{Ca}$ content and canalicular density when assuming a homogenous matrix mineralization.}

Before presenting the experimental results, a brief theoretical consideration estimates the expected correlation between local Ca concentration and local canaliculi density (Can.Dn) for the case of an inactive canalicular network, i.e. it is assumed that canaliculi with a fixed average diameter are permeating a homogenous bone matrix mineralization within an osteon. Since the canalicular porosity increases with an increasing density of the network (Can.Dn) thereby reducing the local Ca content, we expect a negative correlation between these two quantities.

In qBEI measurements, canaliculi are not spatially resolved in contrast to osteocyte lacunae, which are excluded from the evaluation. Consequently, the measured qBEI signal is the signal of mineralized collagen matrix with the Ca content $\mathrm{CaCont}_{\text {Matrix }}$ mixed with the canaliculi filled with an embedding material free of $\mathrm{Ca}\left(\mathrm{CaCont}_{\mathrm{LCN}}=0\right)$. Assuming cylindrical canaliculi with an average diameter Can.Dm, the expected measured CaCont is then,

CaCont $=$ CaCont $_{\text {Matrix }} \cdot\left(1-\pi \cdot\left(\frac{\text { Can.Dm }}{2}\right)^{2} \cdot\right.$ Can. Dn $)$

Eq. (1)

where the definition of Can.Dn as total length of canaliculi per unit volume makes the term in parenthesis equal to the volume fraction of mineralized bone matrix. The relationship between CaCont and Can.Dn is linear with a y-intercept equal to the Ca content of the mineralized matrix, CaCont $_{\text {Matrix, }}$ and with a SLOPE equal to

SLOPE $=\left(\right.$ CaCont $\left._{\text {Matrix }}\right) \cdot \pi \cdot\left(\frac{\text { Can.Dm }}{2}\right)^{2}$

Measurements using qBEI reported a most frequent mineral content CaPEAK of $25.95 \mathrm{wt} \% \mathrm{Ca}$ for femoral midshaft bone of adults ${ }^{(36)}$. In order to correct for the presence of LCN porosity in these measurements, we assume a typical canaliculi density for human femur midshaft bone of 0.76 $\mu \mathrm{m} / \mu \mathrm{m}^{3}{ }^{(18)}$ and a canaliculi diameter of $360 \mathrm{~nm}{ }^{(3)}$, resulting to a matrix mineralization, 
$\mathrm{CaCont}_{\text {Matrix }}$ (i.e., y-intercept) of $28.13 \mathrm{wt} \% \mathrm{Ca}$. Using this value for $\mathrm{CaCont}_{\text {Matrix }}$, the expected SLOPE is estimated to be $-2.9 \mathrm{wt} \% \mathrm{Ca} / \mu \mathrm{m} / \mu \mathrm{m}^{3}$. This theoretical value for the SLOPE serves as baseline for the following experimental data. Taking into account reported variability of Can.Dm between values reported in literature $\left(290 \mathrm{~nm}\right.$ to $\left.440 \mathrm{~nm}{ }^{(32)}\right)$ and variations of the matrix mineralization of $26.39<\mathrm{CaCont}_{\text {Matrix }}<29.86$ (estimated using the full width at half height of the Ca distribution ${ }^{(36)}$, we expect to find SLOPE values between -1.7 and $-4.5 \mathrm{wt} \% \mathrm{Ca} / \mu \mathrm{m} / \mu \mathrm{m}^{3}$ (Fig. 3 - blue area).

Under the assumption that the overwhelming amount of $\mathrm{Ca}$ in bone is present in a mineral phase of bone apatite, it is possible to convert the SLOPE to a change in the amount of Ca per $\mu \mathrm{m}$ of canalicular length as follows: The measured values of the Ca content in $\mathrm{wt} \% \mathrm{Ca}$ can be expressed as a volume fraction of the mineral phase considering the differences in the mass density between the mineral and the organic phase of bone as described in ${ }^{(37)}$. Assuming pure hydroxyapatite with $10 \mathrm{Ca}$ atoms per unit cell within a unit cell volume of $530 \AA^{3}{ }^{(38)}$, approximately $1.89^{*} 10^{10} \mathrm{Ca}$ atoms are found per $\mu \mathrm{m}^{3}$ of pure hydroxyapatite. This estimation is only marginally modified when considering the various chemical substitutions ${ }^{(39)}$ in bone apatite with respect to hydroxyapatite. This conversion of a change in the $\mathrm{Ca}$ content to a change in the number of $\mathrm{Ca}$ atoms per volume, allows us to express the expected SLOPE as $-0.97 \mathrm{fmol} \mathrm{Ca} / \mu \mathrm{m}$ or $5.8 * 10^{8} \mathrm{Ca}$ atoms $/ \mu \mathrm{m}$. The variations in Ca content and Can.Dm as given above result in an upper and lower boundary of 0.58 and $-1.48 \mathrm{fmol} \mathrm{Ca} / \mu \mathrm{m}$, respectively (see fig. $3 \mathrm{~b}$ blue dashed lines).

\section{Measured correlation between Ca content and canalicular density}

Fig. 3a shows a plot of the canalicular density and the Ca content evaluated in around 1000 subvolumes (with edge size $7.37 \mu \mathrm{m}$ ) covering the whole osteon in the femoral cortex (adult 1, see Table 1). This representative data set shows substantial scattering of the data points. A correlative 
analysis results in a highly significant positive correlation (Fig. 3a, red line, $\mathrm{p}<0.0001$, SLOPE $=$ $\left.+4.5 \mathrm{wt} \% \mathrm{Ca} / \mu \mathrm{m} / \mu \mathrm{m}^{3}, \mathrm{R}^{2}=0.15\right)$ indicating increasing $\mathrm{Ca}$ content with increasing canalicular density. For this specific osteon the SLOPE of the linear regression line in Fig 3a corresponds to an increase of $1.67 \mathrm{fmol} \mathrm{Ca}$ per $\mu \mathrm{m}$ of network.

In 62 out of 65 investigated osteons, we found an increase in the local Ca content due to a denser network (median $+1.15 \mathrm{fmol} \mathrm{Ca} / \mu \mathrm{m}$ ). After grouping the osteons, all 6 individuals show significantly higher values than the reference value for bone with homogenous matrix mineralization $(-0.97 \mathrm{fmol} \mathrm{Ca} / \mu \mathrm{m}$, Fig. 3b). Significance also remains preserved when testing versus $0 \mathrm{fmol} \mathrm{Ca} / \mu \mathrm{m}$ (i.e., no change in $\mathrm{Ca}$ due to the network). Median values for the individuals vary between +0.51 and $+1.83 \mathrm{fmol} \mathrm{Ca} / \mu \mathrm{m}$. The small box sizes in Fig. $3 \mathrm{~b}$ indicate that the change in stored Ca per network length is a rather conserved quantity in individuals. 

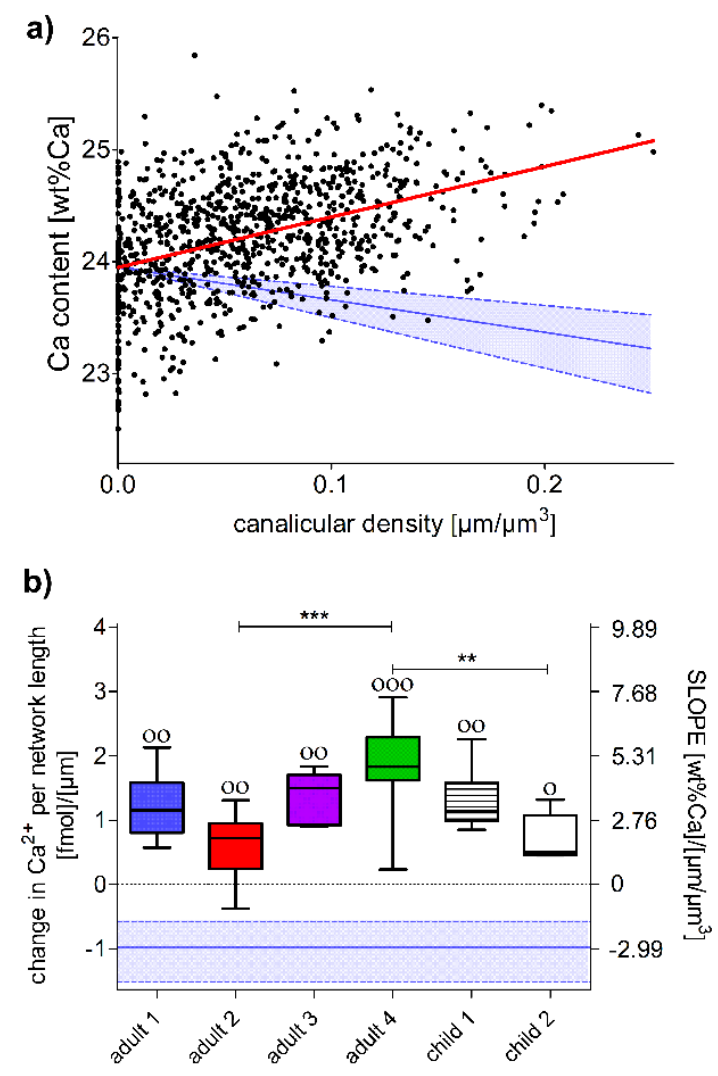

Figure 3: a) Evaluation of the correlation between canalicular density and Ca content within a single osteon of adult 1. Each data point corresponds to a CSLM and qBEI measurement on the same subvolume of edge size $7.37 \mu \mathrm{m}$, where the subvolumes cover the whole cross-sectional area of the osteon (see Fig. 2b) and c)). The red line is the result of a linear regression of the data. The SLOPE obtained for this osteon is $4.5 \mathrm{wt} \% \mathrm{Ca} / \mu \mathrm{m} / \mu \mathrm{m}^{3}$ or $1.67 \mathrm{fmol} \mathrm{Ca}$ per $\mu \mathrm{m}$.

b) Boxplots (line corresponding to median, box to $25 \%-75 \%$ percentile and whiskers to data range) of the change in fmol Ca per $\mu \mathrm{m}$ of network (left y-axis). The values for the $\mathrm{Ca}$ concentrations were derived from the SLOPE values (right y-axis) as described in the text above. Data are based on the evaluation of 65 osteons following the evaluation procedure shown in a) and grouping the osteons according to the 6 individuals (4 adults, 2 children, see Table 1 for number of investigated osteons). Bars indicate significant differences between the individuals (Bonferroni Test). Circles indicate medians significantly different from $-0.97 \mathrm{fmol} / \mu \mathrm{m}(=-2.9$ $\left.\mathrm{wt} \% \mathrm{Ca} /(\mu \mathrm{m} / \mu \mathrm{m})^{3}\right)$, the theoretical reference value for a network within a homogeneously mineralized matrix represented by the blue line in both plots. The blue shaded areas take into account variations of the reference value due to variations in canalicular diameter and matrix mineralization.

\section{Radial dependency of Ca content and canalicular density}


In the majority of the samples we found a systematic dependency of the Ca content and of the canalicular density as a function of the shortest distance of the volume element to the Haversian canal denoted as R. A representative result for a single osteon (adult 4) is shown in Fig. 4a) and Fig. 4c) revealing negative correlations for the $\mathrm{Ca}$ content $(\Delta \mathrm{CaCont} / \Delta \mathrm{R}=-0.01 \mathrm{wt} \% \mathrm{Ca} / \mu \mathrm{m}$, $\left.\mathrm{p}<0.0001, \mathrm{R}^{2}=0.26\right)$ and the Can.Dn $\left(\Delta \mathrm{Can} . \mathrm{Dn} / \Delta \mathrm{R}=-0.00041 / \mu \mathrm{m}^{3}, \mathrm{p}<0.0001, \mathrm{R}^{2}=0.10\right)$ with the distance to the Haversian canal. Using the correlation values of the linear regression to quantify the spatial dependency, statistical analysis confirms significant decreases $(p<0.05)$ for both quantities with the distance to the Haversian canal within all adults except for the Ca content of adult $1(\mathrm{p}=0.1)$ (Fig. $4 \mathrm{~b}, \mathrm{~d})$. In children these radial dependencies are less clear resulting in a significant decrease only of the canalicular density with the distance to the Haversian canal in both children (Fig. 4 b,d).

The observation of radial dependencies raises the questions if the increase in the amount of Ca per network length reported above is a consequence of these radial dependencies. To address this point in Fig. 4 the values of the radial dependencies of the single osteons are plotted for both quantities. Most of the osteons exhibit negative correlations of both quantities with the distance of the Haversian canal (lower left quadrant in Fig. 5), but there is no clear trend that more negative values in one quantity are correlated with more negative values in the other. Even more, this lack of correlation between the two radial dependencies is demonstrated in 8 out of the 65 osteons, in which the two quantities have an opposite sign. Specifically, a positive radial dependency of the Ca content is often accompanied by a negative radial dependency in the canalicular density (upper left quadrant in Fig. 5). A check confirmed that all of the osteons in this quadrant exhibit an increase in the amount of Ca per network length. Consequently, this increase cannot be explained by a radial dependency of the two quantities only, and thus, more local correlations must be assumed. 
a)

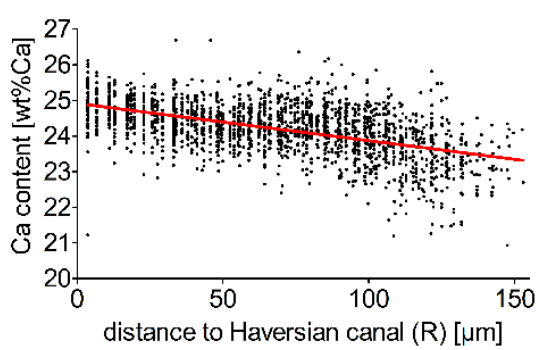

c)

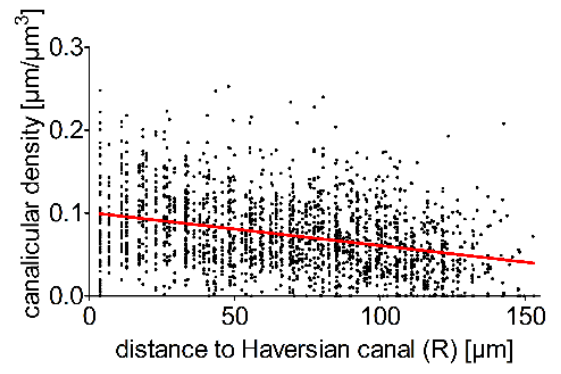

b)

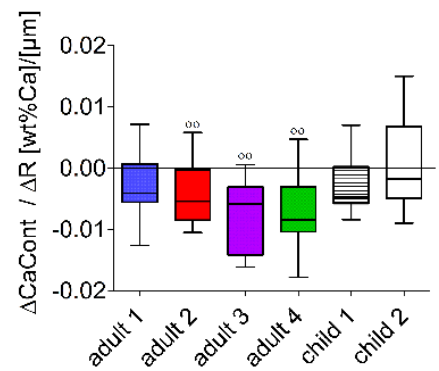

d)

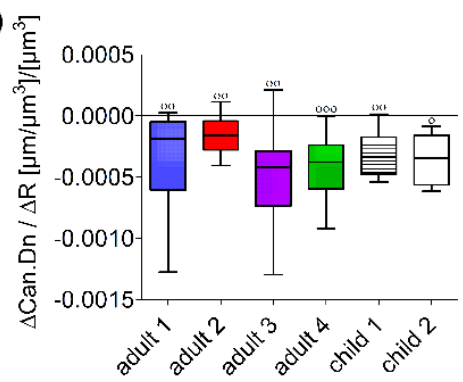

Figure 4 a), c): Radial dependency of the Ca content (a) and the canalicular density (c) with the shortest distance to the Haversian canal for one representative osteon of adult 4. Each data point corresponds to the measurement outcome within one voxel as depicted in Fig. 2 d) and b), c), respectively. The red line is the result of a linear regression of all data points. (b), (d) Boxplots (line corresponding to median, box to $25 \%-75 \%$ percentile and whiskers to data range) of the evaluation of the radial dependencies for all osteons, where values from the linear regression were obtained like shown in a) and c). Circles indicate significant differences vs. 0 (Wilcoxon signed-rank test), bars differences between the individuals (Bonferroni Test).

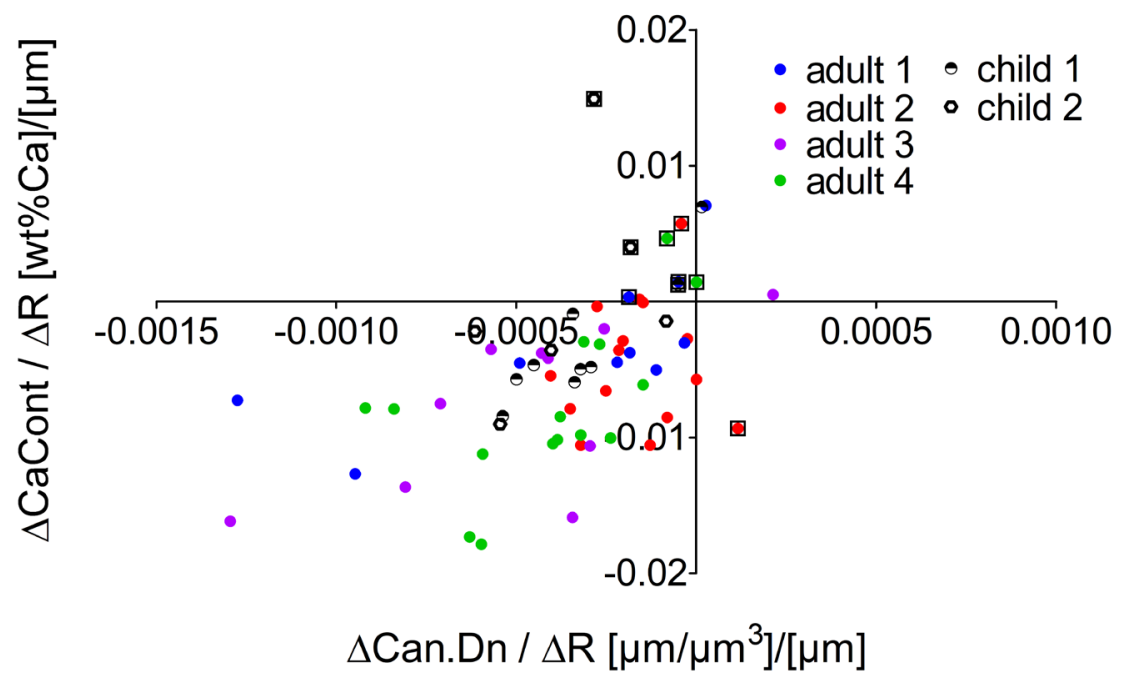

Figure 5: Radial dependency of the $\mathrm{Ca}$ content $(\Delta \mathrm{CaCont} / \Delta \mathrm{R})$ and canalicular density $(\Delta \mathrm{Can} . \mathrm{Dn} / \Delta \mathrm{R}$ ) as evaluated by a linear regression (see Fig. $4 \mathrm{a}, \mathrm{c}$ ), $\mathrm{R}$ denoting the shortest distance to the Haversian canal. The majority of osteons show a negative correlation for both 
Can.Dn and Ca content with the distance to the Haversian canal (lower left quadrant). However, data points in the upper left and lower right quadrant (denoted by squares) exhibit opposite signs in the radial dependencies of the two quantities.

\section{Average Ca content and canalicular density}

Fig. 6 shows the Ca content and canalicular density averaged over the osteons measured in the 6 individuals. We found median values for the Ca content between 25.73 and $21.05 \mathrm{wt} \% \mathrm{Ca}$ (Fig. 6a). Median canalicular density varies between 0.07 and $0.14 \mu \mathrm{m} / \mu \mathrm{m}^{3}$ (Fig 6b). Even though samples exhibiting the lowest $\mathrm{Ca}$ content and highest canalicular density originate from children, it should be noted that the sample number is too low to make a general statement regarding the age dependency at this point.
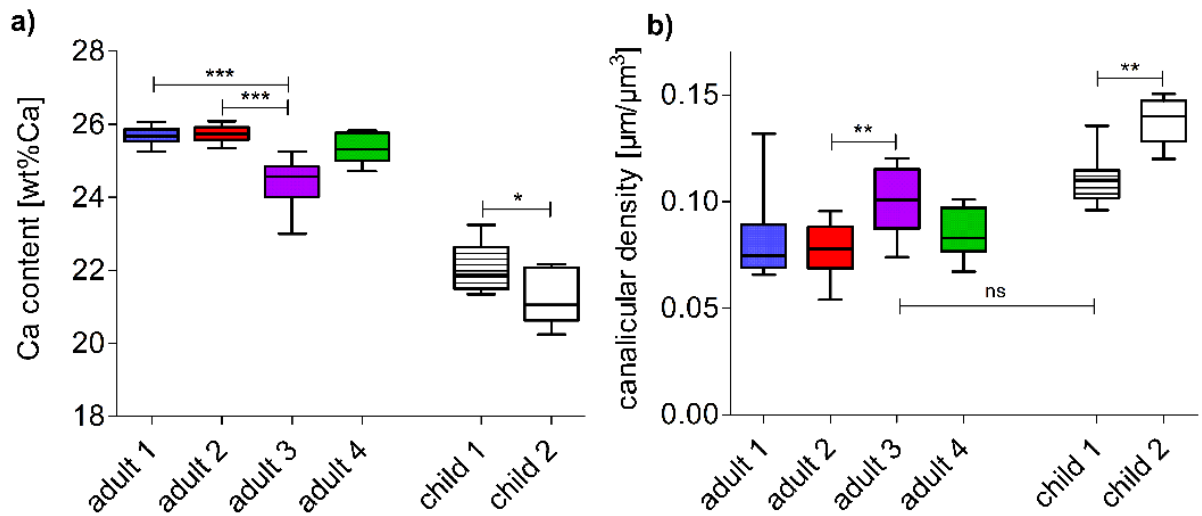

Figure 6: Boxplots (line corresponding to median, box to $25 \%-75 \%$ percentile and whiskers to data range) of the Ca content (a) and canalicular density (b) considering all the osteons in a single individual. Bars indicate differences between individuals of the same age group (Bonferroni Test). Comparisons of individuals of different age groups revealed to be significant if not indicated differently.

\section{DISCUSSION}

In this paper, we evaluate two linked structural quantities within osteons of human femoral bone: the lacunar-canalicular network ( $\mathrm{LCN}$ ) density (Can.Dn - referring to the length of the canaliculi per unit volume) and the local calcium concentration (Ca content). To obtain robust results, we 
suggest -based on the network geometry- that the evaluation volume for canalicular density should not be smaller than $400 \mu \mathrm{m}^{3}$ as used in this study. The same spatial resolution was applied for both experimental methods to study the correlation of the local Ca content and Can.Dn.

A positive correlation (SLOPE) between Ca content and canalicular density is found in 62 out of 65 osteons. This can be expressed as an increase in the amount of Ca per network length (fmol $\mathrm{Ca} / \mu \mathrm{m})$. We found median values between $+0.51 \mathrm{fmol} \mathrm{Ca} / \mu \mathrm{m}$ and $+1.83 \mathrm{fmol} \mathrm{Ca} / \mu \mathrm{m}$. The finding that the $\mathrm{Ca}$ content of bone is positively correlated with length of the network is even more remarkable since theoretical considerations (which estimate the microporosity of the LCN and the resulting reduction of the $\mathrm{Ca}$ content) predict a decrease of the amount of Ca due to the $\mathrm{LCN}$ by $0.97 \mathrm{fmol} / \mu \mathrm{m}$. None of the investigated osteons exhibit values in the expected range. Consequently, the assumption of a bone matrix with a homogenous mineral content only infiltrated by the canalicular network leads to contradictions to experimental findings.

An increase in the amount of stored $\mathrm{Ca}$ with network length implies that regions of dense LCN exhibit elevated $\mathrm{Ca}$ concentrations. This consideration assumes an interaction between the LCN and mineralized matrix and therefore may relates to the questions of osteolytic osteolysis and pericanalicular remodeling. The observed effect can be explained by two hypotheses: (i) Regions of high canalicular density exhibit overall a higher $\mathrm{Ca}$ content, because of improved transport properties by a dense LCN and an efficient supply with mineral precursors. In other words, the density of the canalicular network would influence the kinetics of secondary mineralization (as defined in reference ${ }^{(26)}$ ); (ii) Pericanalicular regions exhibit locally bone of increased Ca content overcompensating the porosity effect of the LCN in the obtained measurements.

Reznikov et al. observed that canaliculi are mainly located in narrow regions of loose disordered collagen packing in humans ${ }^{(25,40)}$. Such disordered arrangement of collagen fibrils could leave 
more space for mineral incorporation explaining a local hypermineralization. These findings support hypothesis (ii) suggesting confined regions around canaliculi (influencing zone) which can be more or less loaded with mineral. According to our results one $\mu \mathrm{m}$ of network length is associated with an average increase of $1.15 \mathrm{fmol}$ of $\mathrm{Ca}\left(6.9 * 10^{8}\right.$ atoms $\left./ \mu \mathrm{m}\right)$. This value describes the LCN-related increase of mineral density. This finding of a systemic storage of $\mathrm{Ca}$ in relation with the LCN provides information over a large (osteonal) length scale and therefore complements the work of Hesse et al. who were employing a high resolution method to map the direct vicinity of the canaliculi in osteonal and interstitial bone ${ }^{(3)}$. They found a spatial dependency of the mineral content on the distance to the canaliculus with the highest mineral content close to the canaliculus. Bringing together these two findings characterizing mineral heterogeneities in bone on two different length scales, we suggest two simple geometrical models to fathom the consequences of the obtained quantitative correlation between network density and Ca content. Both models assume typical values for the diameter of canaliculi and matrix mineralization and a hypermineralized cylindrical influencing zone around each canaliculi in order to fit the measured data (increase of $1.15 \mathrm{fmol} \mathrm{Ca} / \mu \mathrm{m}$ ). The models differ in the assumption of the spatial gradient of the Ca content around the canaliculi (details of the models are provided in the Appendix). Despite different model assumptions both models agree with a width of the influencing zone, which is roughly 1.5 times the diameter of canaliculi (see Appendix).

The experiments combined with theoretical considerations suggest that the vicinity of the canaliculi could be used by the osteocytes as easily accessible source of mineral. Osteocytes would then change the pericanalicular matrix by depositing or resorbing mineral depending on homeostatic mineral need and the additional Ca loading of the network could be interpreted as a filling quantity 
of a mineral reservoir. As the observed values of the additional Ca loading show relatively low variations within the osteons of a single individual, but show inter-individual differences, we conjecture that the obtained results are characteristic for the individual mirroring systemic conditions of the organism.

There is a wide range of unknown factors like age, diseases, hormonal balance, dietary and sport activity potentially influencing bone turnover and therefore mineral content. For example, the investigated adult bone samples originate all from females at an age close to the onset of menopause. Since hormonal changes associated with menopause increase the rate of bone turnover, also the mineral content is impacted ${ }^{(26)}$. Little is known about the influence of these factors on the LCN properties. The larger dimensions of osteocyte lacunae imply that most studies focused on changes in lacunar density and shape of the lacunae, nevertheless much progress is made in the description of the $\mathrm{LCN}^{(41,42)}$.

Concerning the canalicular density and its network, a reduced connectivity is reported for osteoporotic and osteoarthritic bone. In high fat-fed diabetic mice a higher mean node degree was found accompanied with a decreased average edge length ${ }^{(43)}$. Vashishth et al. found a decrease of the lacuna density in human cortical bone with age ${ }^{(44)}$. Also a mouse model indicates impaired network connectivity and decreased cell volume during aging ${ }^{(45)}$ what is consistent with findings in humans ${ }^{(7)}$. Aging and hormonal changes might also influence the correlations reported in this study. Future projects on bones of patients with a known hormonal status will show its influence on the relation between mineral content and LCN density.

While an additional Ca loading of the network is a general characteristic of the investigated osteons, the observed substantial scatter in the correlation plots (Fig. 3a) requires an extra consideration. 
Contributors to this scatter are experimental limitations like the finite measurement time of qBEI (46) and limitations in the spatial resolution, in particular the depth (z-direction), in confocal microscopy. Furthermore, information depth and the alignment of the evaluation grid onto two different instruments may induce some errors, and consequently, the evaluated bone volumes are not fully identical. However, we assume that most of the observed scattering is due to structural heterogeneities, e.g. local variations in the diameter of the canaliculi and the width of the influencing zone of the mineral reservoir. As the measured differences in mineral content are relatively small compared to what is observed in the regular mineralization process, it should be noted that using a backscatter electron technique, typical artifacts that might occur with other methods can be ruled out as systematic errors. For example, when using micro-computer tomographic based methods, beam hardening effects might adulterate the interpretation of material density and possible artifacts need to be taken into consideration at sharp interfaces of different material densities when using a phase contrast setup ${ }^{(47,48)}$.

Besides the finding that the local mineral content is closely linked to the LCN properties, two further results deserve attention. First, the lowest $\mathrm{Ca}$ content values and highest canalicular densities were observed in bone originating from children (Fig 6a). This is in line with reports for trabecular bone, where the bone mineralization density distribution in children was found to be shifted to lower Ca values ${ }^{(49)}$ compared to the trabecular reference BMDD for healthy adults ${ }^{(50)}$. While a change of the mineral content with tissue age is reasonable considering the mineralization process, less is known about possible differences in the density of the canalicular network between children and adults. Due to the limited sample number in the presented study no general statement on the age dependency of the parameters can be made. 
Second, canalicular density and $\mathrm{Ca}$ content were found to decrease with the distance to the Haversian canal. These radial dependencies are consistent with reports in the literature describing a radial decrease of the network density in human osteons ${ }^{(18)}$ and electron dense material at regions close to the Haversian canals ${ }^{(51)}$. Latter appears to be contradictious with the intensely investigated mineralization kinetics linking tissue age with degree of mineralization ${ }^{(26,52-54)}$ describing ongoing mineral crystal growth with tissue age. Since in the formation of the osteon the bone close to the cement line is formed first and close to the Haversian canal latest, the expected spatial gradient is exactly the opposite to what was measured in this study. Martin et al. also reported higher mineral content close to the Haversian canal showing that this effect cannot be explained with simple mineral diffusion models ${ }^{(51)}$. Our explanation is that due to an increased canalicular density closer to the Haversian canal and the correlation between canalicular density and $\mathrm{Ca}$ content, the tissue age effect might be overcompensated by the LCN-related increase of mineral content causing a negative mineralization gradient moving from the Haversian canal towards the cement line.

In summary, this study provides evidence for the existence of a mineral reservoir around the canaliculi, which can be actively manipulated by osteocytes for a fast-access contribution to mineral homeostasis. This conclusion is based on a positive spatial correlation between canalicular density and $\mathrm{Ca}$ content in human osteons. Differences in the mineral load of the reservoir zones between the individuals indicate individual-specific variations in the utilization of the mineral reservoir. We hypothesize that the amount of mineral stored in the reservoir is a dynamic parameter, which can adapt according to the needs of the organism. A crucial test of this hypothesis would be studies on individuals suffering from diseases of mineral deficiencies and corresponding mouse models. We expect that such studies will be of important help for a correct interpretation of the parameter introduced in this work, the network loading with calcium. In addition to this, we 
propose that high-resolution experimental techniques should be harnessed for a closer investigation of the pericanalicular and perilacunar regions. A spatial resolution of down to $10 \mathrm{~nm}$ is, for example, achievable with 3D backscattered electron microscopy facilitated by ion beam surface removal (FIB/SEM).

\section{Acknowledgements}

The authors thank Petra Keplinger, Sonja Lueger, Phaedra Messmer, and Daniela Gabriel for sample preparation at the Ludwig Boltzmann Institute of Osteology, Vienna, Austria. The research presented here was supported by a grant from the German Federal Ministry of Education and Research (Grant No. 01EC1402D) and by the AUVA (Research Funds of the Austrian Workers Compensation Board, Austria) and the WGKK (Viennese sickness insurance funds, Austria). 


\section{REFERENCES}

1. $\quad$ Bonewald LF. The amazing osteocyte. J Bone Miner Res. Feb 2011;26(2):229-38.

2. Schaffler MB, Cheung WY, Majeska R, Kennedy O. Osteocytes: master orchestrators of bone. Calcif Tissue Int. Jan 2014;94(1):5-24. Epub 2013/09/18.

3. Hesse B, Varga P, Langer M, Pacureanu A, Schrof S, Mannicke N, et al. Canalicular network morphology is the major determinant of the spatial distribution of mass density in human bone tissue: evidence by means of synchrotron radiation phase-contrast nano-CT. J Bone Miner Res. Feb 2015;30(2):346-56.

4. Kerschnitzki M, Wagermaier W, Roschger P, Seto J, Shahar R, Duda GN, et al. The organization of the osteocyte network mirrors the extracellular matrix orientation in bone. J Struct Biol. Feb 2011;173(2):303-11.

5. Genthial R, Beaurepaire E, Schanne-Klein MC, Peyrin F, Farlay D, Olivier C, et al. Label-free imaging of bone multiscale porosity and interfaces using third-harmonic generation microscopy. Sci RepUk. Jun 13 2017;7.

6. Knothe Tate ML, Knothe U. An ex vivo model to study transport processes and fluid flow in loaded bone. J Biomech. Feb 2000;33(2):247-54. Epub 2000/02/01.

7. Ashique AM, Hart LS, Thomas CDL, Clement JG, Pivonka P, Carter Y, et al. Lacunar-canalicular network in femoral cortical bone is reduced in aged women and is predominantly due to a loss of canalicular porosity. Bone Rep. Dec 2017;7:9-16. Epub 2017/07/29.

8. Milovanovic P, Zimmermann EA, Hahn M, Djonic D, Puschel K, Djuric M, et al. Osteocytic Canalicular Networks: Morphological Implications for Altered Mechanosensitivity. Acs Nano. Sep 2013;7(9):7542-51.

9. Kerschnitzki M, Kollmannsberger P, Burghammer M, Duda GN, Weinkamer R, Wagermaier W, et al. Architecture of the osteocyte network correlates with bone material quality. J Bone Miner Res. Aug 2013;28(8):1837-45.

10. Repp F, Kollmannsberger P, Roschger A, Berzlanovich A, Gruber GM, Roschger P, et al. Coalignment of osteocyte canaliculi and collagen fibers in human osteonal bone. J Struct Biol. Aug 012017. Epub 2017/08/06.

11. Fritton SP, Weinbaum S. Fluid and Solute Transport in Bone: Flow-Induced Mechanotransduction. Annu Rev Fluid Mech. 2009;41:347-74.

12. Klein-Nulend J, Sterck JG, Semeins CM, Lips P, Joldersma M, Baart JA, et al. Donor age and mechanosensitivity of human bone cells. Osteoporos Int. 2002;13(2):137-46. Epub 2002/03/23.

13. Knothe Tate ML. "Whither flows the fluid in bone?" An osteocyte's perspective. J Biomech. Oct 2003;36(10):1409-24.

14. Teti A, Zallone A. Do osteocytes contribute to bone mineral homeostasis? Osteocytic osteolysis revisited. Bone. Jan 2009;44(1):11-6.

15. Wysolmerski JJ. Osteocytic osteolysis: time for a second look? Bonekey Rep. Dec 5 2012;1:229. Epub 2012/01/01.

16. Belanger LF. Osteocytic osteolysis. Calcif Tissue Res. Aug 11 1969;4(1):1-12. Epub 1969/08/11.

17. Buenzli PR, Sims NA. Quantifying the osteocyte network in the human skeleton. Bone. Jun 2015;75:144-50.

18. Repp F, Kollmannsberger P, Roschger A, Kerschnitzki M, Berzlanovich A, Gruber GM, et al. Spatial heterogeneity in the canalicular density of the osteocyte network in human osteons. Bone Rep. Jun 2017;6:101-8.

19. Nango N, Kubota S, Hasegawa T, Yashiro W, Momose A, Matsuo K. Osteocyte-directed bone demineralization along canaliculi. Bone. Mar 2016;84:279-88. 
20. Qing H, Ardeshirpour L, Pajevic PD, Dusevich V, Jahn K, Kato S, et al. Demonstration of osteocytic perilacunar/canalicular remodeling in mice during lactation. J Bone Miner Res. May 2012;27(5):1018-29.

21. Fowler TW, Acevedo C, Mazur CM, Hall-Glenn F, Fields AJ, Bale HA, et al. Glucocorticoid suppression of osteocyte perilacunar remodeling is associated with subchondral bone degeneration in osteonecrosis. Sci Rep. Mar 22 2017;7:44618. Epub 2017/03/23.

22. Jahn K, Kelkar S, Zhao H, Xie Y, Tiede-Lewis LM, Dusevich V, et al. Osteocytes Acidify Their Microenvironment in Response to PTHrP In Vitro and in Lactating Mice In Vivo. J Bone Miner Res. May 042017.

23. Bach-Gansmo FL, Wittig NK, Bruel A, Thomsen JS, Birkedal H. Immobilization and long-term recovery results in large changes in bone structure and strength but no corresponding alterations of osteocyte lacunar properties. Bone. Oct 2016;91:139-47. Epub 2016/07/13.

24. Sharma D, Ciani C, Marin PAR, Levy JD, Doty SB, Fritton SP. Alterations in the osteocyte lacunarcanalicular microenvironment due to estrogen deficiency. Bone. Sep 2012;51(3):488-97.

25. Reznikov N, Shahar R, Weiner S. Bone hierarchical structure in three dimensions. Acta Biomater. Sep 2014;10(9):3815-26.

26. Roschger $P$, Paschalis EP, FratzI $P$, Klaushofer $K$. Bone mineralization density distribution in health and disease. Bone. Mar 2008;42(3):456-66.

27. Roschger P, Fratzl P, Eschberger J, Klaushofer K. Validation of quantitative backscattered electron imaging for the measurement of mineral density distribution in human bone biopsies. Bone. Oct 1998;23(4):319-26.

28. Roschger P, Plenk H, Klaushofer K, Eschberger J. A New Scanning Electron-Microscopy Approach to the Quantification of Bone-Mineral Distribution - Backscattered Electron Image Grey-Levels Correlated to Calcium K-Alpha-Line Intensities. Scanning Microscopy. Mar 1995;9(1):75-88.

29. Repp F. TINA network analysis tool. 2017.

30. Kollmannsberger $\mathrm{P}$, Kerschnitzki M, Repp F, Wagermaier W, Weinkamer R, Fratzl P. The small world of osteocytes: connectomics of the lacuno-canalicular network in bone. New J Phys. Jul 18 2017;19.

31. Marr D, Hildreth E. Theory of edge detection. Proc R Soc Lond B Biol Sci. Feb 29 1980;207(1167):187-217.

32. Varga P, Hesse B, Langer M, Schrof S, Mannicke N, Suhonen H, et al. Synchrotron X-ray phase nanotomography-based analysis of the lacunar-canalicular network morphology and its relation to the strains experienced by osteocytes in situ as predicted by case-specific finite element analysis. Biomech Model Mechanobiol. Apr 2015;14(2):267-82.

33. Roschger A, Gamsjaeger S, Hofstetter B, Masic A, Blouin S, Messmer P, et al. Relationship between

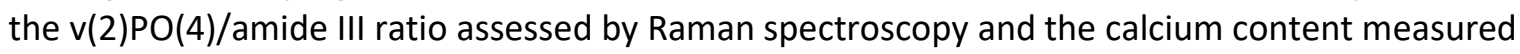
by quantitative backscattered electron microscopy in healthy human osteonal bone. J Biomed Opt. Jun 2014;19(6).

34. Goldstein J, Newbury, D.E., Joy, D.C., Lyman, C.E., Echlin, P., Lifshin, E., Sawyer, L., Michael, J.R. . Scanning Electron Microscopy and X-ray Microanalysis. New York: Springer Science + Business Media New York; 2003.

35. Hassler N, Roschger A, Gamsjaeger S, Kramer I, Lueger S, van Lierop A, et al. Sclerostin deficiency is linked to altered bone composition. J Bone Miner Res. Oct 2014;29(10):2144-51.

36. Buenzli PR, Lerebours C, Roschger A, Roschger P, Weinkamer R. Late stages of mineralization and their signature on the bone mineral density distribution. Connective Tissue Research. 2018;59:7480.

37. Zizak I, Roschger P, Paris O, Misof BM, Berzlanovich A, Bernstorff S, et al. Characteristics of mineral particles in the human bone/cartilage interface. J Struct Biol. Mar 2003;141(3):208-17. 
38. Zeglinski J, Nolan M, Bredol M, Schatte A, Tofail SAM. Unravelling the specific site preference in doping of calcium hydroxyapatite with strontium from ab initio investigations and Rietveld analyses. Phys Chem Chem Phys. 2012;14(10):3435-43.

39. Wopenka B, Pasteris JD. A mineralogical perspective on the apatite in bone. Mat Sci Eng C-Bio S. Apr 28 2005;25(2):131-43.

40. Reznikov N, Shahar R, Weiner S. Three-dimensional structure of human lamellar bone: the presence of two different materials and new insights into the hierarchical organization. Bone. Feb 2014;59:93-104. Epub 2013/11/12.

41. Schneider P, Meier M, Wepf R, Muller R. Towards quantitative 3D imaging of the osteocyte lacunocanalicular network. Bone. Nov 2010;47(5):848-58.

42. Mader KS, Schneider P, Muller R, Stampanoni M. A quantitative framework for the 3D characterization of the osteocyte lacunar system. Bone. Nov 2013;57(1):142-54.

43. Mabilleau G, Perrot R, Flatt PR, Irwin N, Chappard D. High fat-fed diabetic mice present with profound alterations of the osteocyte network. Bone. Sep 2016;90:99-106.

44. Vashishth D, Verborgt O, Divine G, Schaffler MB, Fyhrie DP. Decline in osteocyte lacunar density in human cortical bone is associated with accumulation of microcracks with age. Bone. Apr 2000;26(4):375-80.

45. Tiede-Lewis LM, Xie YX, Hulbert MA, Campos R, Dallas MR, Dusevich V, et al. Degeneration of the osteocyte network in the C57BL/6 mouse model of aging. Aging-Us. Oct 2017;9(10):2187-+.

46. Lukas C, Kollmannsberger P, Ruffoni D, Roschger P, Fratzl P, Weinkamer R. The Heterogeneous Mineral Content of Bone-Using Stochastic Arguments and Simulations to Overcome Experimental Limitations. J Stat Phys. Jul 2011;144(2):316-31.

47. Brooks RA, Dichiro G. Beam Hardening in X-Ray Reconstructive Tomography. Phys Med Biol. 1976;21(3):390-8.

48. Langer M, Pacureanu A, Suhonen H, Grimal Q, Cloetens P, Peyrin F. X-Ray Phase Nanotomography Resolves the 3D Human Bone Ultrastructure. Plos One. Aug 29 2012;7(8).

49. Fratzl-Zelman N, Roschger P, Misof BM, Pfeffer S, Glorieux FH, Klaushofer K, et al. Normative data on mineralization density distribution in iliac bone biopsies of children, adolescents and young adults. Bone. Jun 2009;44(6):1043-8.

50. Roschger P, Gupta HS, Berzlanovich A, Ittner G, Dempster DW, Fratzl P, et al. Constant mineralization density distribution in cancellous human bone. Bone. Mar 2003;32(3):316-23. Epub 2003/04/02.

51. Martin B. Mathematical model for the mineralization of bone. J Orthop Res. May 1994;12(3):37583. Epub 1994/05/01.

52. Fratzl P, Fratzlzelman N, Klaushofer K, Vogl G, Koller K. Nucleation and Growth of Mineral Crystals in Bone Studied by Small-Angle X-Ray-Scattering. Calcified Tissue Int. Jun 1991;48(6):407-13.

53. Gamsjaeger S, Masic A, Roschger P, Kazanci M, Dunlop JWC, Klaushofer K, et al. Cortical bone composition and orientation as a function of animal and tissue age in mice by Raman spectroscopy. Bone. Aug 2010;47(2):392-9.

54. Ruffoni $D$, Fratzl $P$, Roschger $P$, Klaushofer $K$, Weinkamer R. The bone mineralization density distribution as a fingerprint of the mineralization process. Bone. May 2007;40(5):1308-19. Epub 2007/03/06. 


\section{APPENDIX}

To interpret the obtained values for the increase of stored $\mathrm{Ca}$ due to the network in terms of the hypermineralized region around the canaliculi, we explore two simplified models (Fig. 7): focusing on adult individuals, cylindrical canaliculi of diameter $360 \mathrm{~nm}$ are surrounded by an influencing zone of increased $\mathrm{Ca}$ content compared to the matrix Ca content far away from the canaliculi being $25.22 \mathrm{wt} \% \mathrm{Ca}$ calculated as average intercept of all adult osteons. In the first model we assume a fixed size of the influencing zone of $0.5 \mu \mathrm{m}$ (Fig. 7b) and ask how large the amount of hypermineralization must be to explain the increase of $1.15 \mathrm{fmol}$ Ca per $\mu \mathrm{m}$ of network. The result is that a homogeneous $\mathrm{Ca}$ content of $29.45 \mathrm{wt} \% \mathrm{Ca}$ in the influencing zone, i.e. an increase of $16.7 \%$ compared to the matrix $\mathrm{Ca}$ content, would be sufficient to explain measurements. An increase of $16.7 \%$ seems reasonable and in accordance with the bone structure based on the natural variability of the mineral content as characterized by the bone mineral density distribution ${ }^{(36)}$. In the second model (Fig. 7c) we assume an exponentially decreasing Ca content around the canaliculi starting at the canaliculus surface with the maximal mineral content value of $39.86 \mathrm{wt} \% \mathrm{Ca}$ (corresponding to pure hydroxyapatite) and ending at the matrix $\mathrm{Ca}$ content of $25.22 \mathrm{wt} \% \mathrm{Ca}$ far away from the canaliculi. Here the question to answer is how fast the spatial decrease of Ca content must be to explain the experimental SLOPE value. The answer is that the width of the influencing zone must be $0.61 \mu \mathrm{m}$ as characterized by a $90 \%$ decrease of the exponential function (Fig. 7c). A gradual decay of mineral content is consistent with what was reported before ${ }^{(3)}$. 

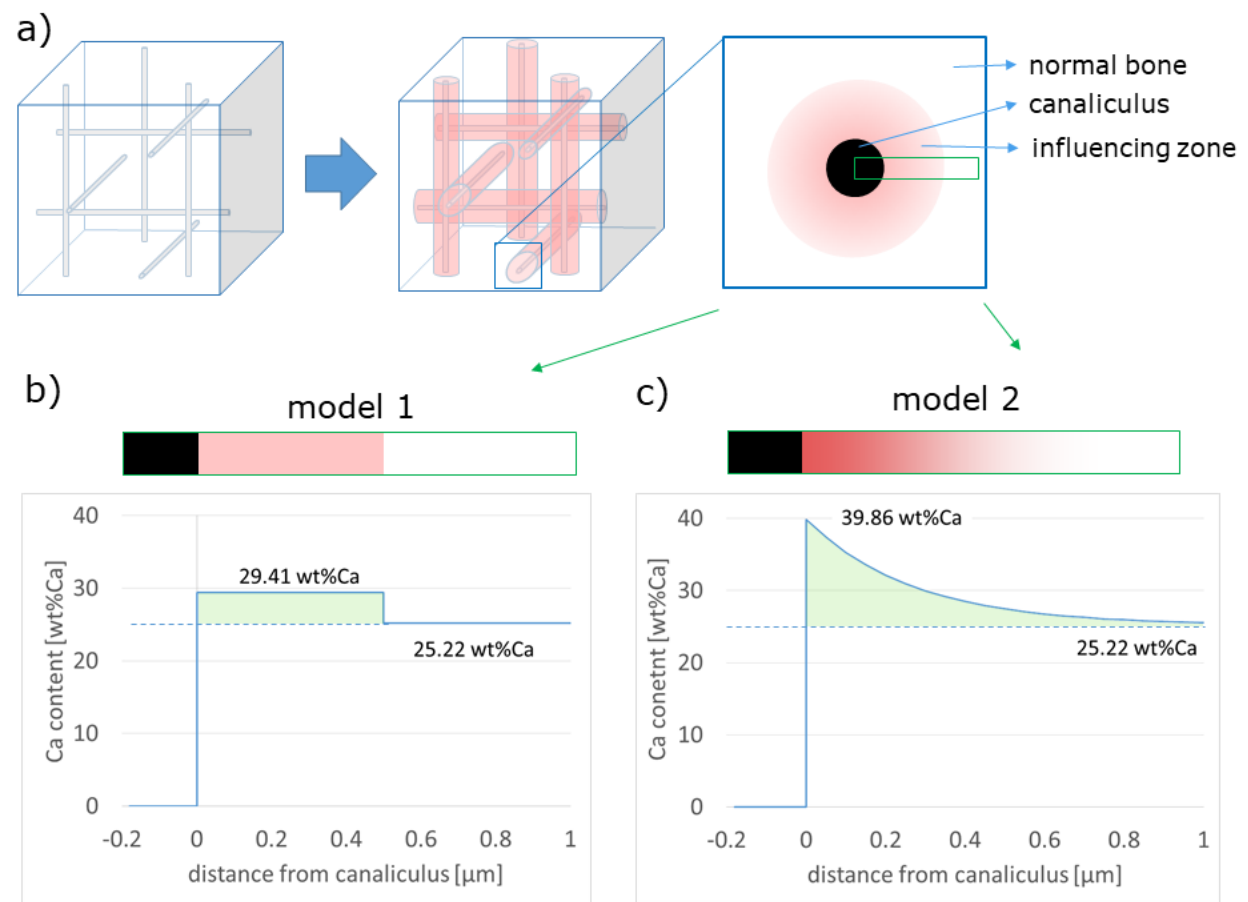

Figure 7: (a) Two simple geometrical models of the canalicular network as cylindrical canals (black/gray) of $360 \mathrm{~nm}$ diameter surrounded by an influencing zone of hypermineralization (red) used for the interpretation of the measured increase of 1.15 fmol Ca per $\mu \mathrm{m}$ of network. (b) In the first model we assume a fixed width of the influencing zone $(0.5 \mu \mathrm{m})$ and calculate the amount of hypermineralization necessary within the influencing zone to be in agreement with experiments (result: $29.45 \mathrm{wt} \% \mathrm{Ca}$ ). (c) In the second model an exponential decay of the $\mathrm{Ca}$ content is assumed and the distance of $90 \%$ reduction of the exponential function is calculated to characterize the width of the influencing zone (result: $0.61 \mu \mathrm{m}$ ).

Areas highlighted in green correspond to the mineral loading of the reservoir zone. 